\title{
A Secondary Coulomb Machine Activating Core-Collapse Supernova Displays
}

\author{
Bradley S. Meyer* \\ Department of Physics and Astronomy, Clemson University, Clemson, SC 29634-0978, USA \\ E-mail: mbradle@clemson.edu

\section{Donald D. Clayton} \\ Department of Physics and Astronomy, Clemson University, Clemson, SC 29634-0978, USA
}

\begin{abstract}
We present an uncommon description of an energy transfer process in core-collapse supernovae: namely, a gravitational machine that increases Coulomb energy within nuclei via silicon burning. Later, excess of that Coulomb energy is returned by weak nuclear decays (EC and beta+). Those decays energize several observable quantities: (1) gamma-ray lines, (2) X-ray luminosity, (3) free chemical energy, (4) optical light curves. The delay of the return energy transfer is essential for visibility of these activations. These secondary displays have rich literatures; but expressing them as observables of a supernova machine, whose action can be summarized as gravitational compression $\rightarrow$ Coulomb nuclear energy increase $\rightarrow$ release of excess of that Coulomb nuclear energy by electroweak decays $\rightarrow$ supernova displays, is novel.
\end{abstract}

XIII Nuclei in the Cosmos,

7-11 July, 2014

Debrecen, Hungary

\footnotetext{
*Speaker.
} 


\section{Introduction}

Four important observable aspects of core-collapse supernovae (SNII) are made visible by radioactive decay of nuclei produced in silicon burning in the inner layers of the exploding star: 1) gamma-ray lines emitted after decay [1,2], 2) continuous spectrum of X rays leaving the SN surface $[3,4], 3)$ non-thermal species made visible in the $\mathrm{SN}$ atmosphere, primarily $\mathrm{He}^{+}$and free $\mathrm{C}$ atoms $[5,6]$, and 4) the supernova light curve after the first month (in SN1987A the exponential light curve radiated $10^{49} \mathrm{ergs}$ from $0.07 M_{\odot}$ of ${ }^{56} \mathrm{Ni}$ nuclei [7]). These four topics allude to a significant portion of supernova research, and the mechanism that makes them observable is clearly important for astrophysics. We present a new way of visualizing that mechanism as "the secondary machine" in core-collapse SN.

A supernova machine is a device that makes use of prompt gravitational work to release energy to overlying SN regions where that energy is capable of causing observable phenomena. We distinguish between two SNII machines. The primary SN machine converts gravitational work into energy that is transported by neutrinos or advection to the SN mantle. That energy causes its explosive ejection. This machine acts promptly, but it is not fully understood (e.g., [8, 9]). The secondary SN machine creates excess Coulomb energy within nuclei by gravitationally-caused compression of the Si shell resulting in larger $Z=N$ nuclei. That excess energy is released only later after considerable expansion and thinning, and that delayed energy release emits non-thermal energy into the mantle. The release of excess Coulomb energy is delayed by long weak lifetimes for electron capture (EC) and $\beta+$ decays, which renders that energy observable, producing phenomena that occur months after core collapse. Without considerable delay, returned energy from nuclei would simply be absorbed into the opaque thermal density of the supernova interior, so that the four activations noted above would not be observable.

The role of radioactivity in exciting astronomical observables has been known for decades, but we believe the concept of the secondary machine helps in delineating the flow of energy in such a complex system as an exploding massive star and in understanding how supernova, in the large, may be viewed. While similar energy storage and release occurs in thermonuclear supernovae (explosions of white dwarf stars), we focus on core-collapse supernovae where the action seems more "machine like".

\section{Silicon Burning}

The secondary machine operates through the action of silicon burning, which occurs in a massive star when the ${ }^{28} \mathrm{Si}$-rich layer in the star is compressed. The compression occurs either by simple gravitational contraction or by the shock wave that rebounds when matter supersonically falls onto the neutron star during stellar collapse. The resulting shock wave is launched by gravitational collapse, so the compression and heating of overlying mantle by that shock wave is also a "gravitationally caused compression".

To model silicon burning in an exploding massive star, we use a simple SNII model [10]. This model makes use of the fact that matter behind the supernova shock is well approximated as homogeneous distribution of radiation-dominated matter [11]. As a result, we treat post-shock material in a spherically symmetric star as an isothermal ball. This provides the necessary constraint on the 


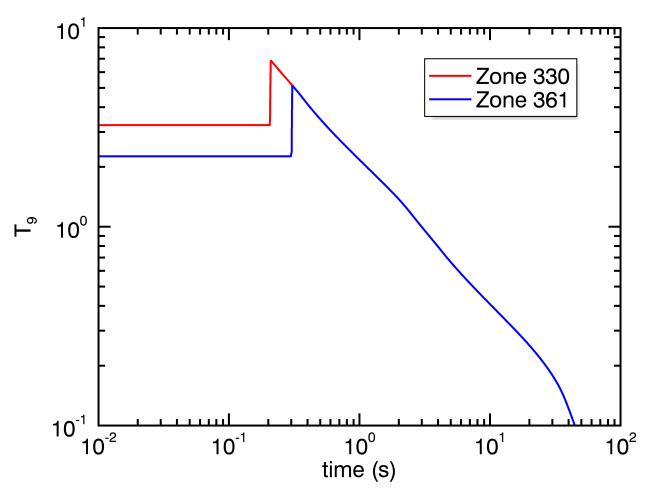

(a)

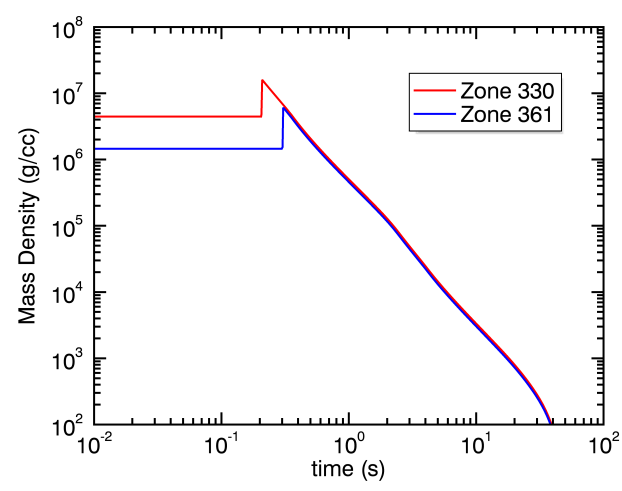

(b)

Figure 1: Time dependence of the (a) temperature $T_{9}=T / 10^{9} \mathrm{~K}$ and (b) mass density in zones 330 and 361 in our simple SNII model.

post shock temperature to solve the Rankine-Hugoniot conditions, given the pre-shock thermodynamic conditions and abundances. In what follows, we apply this simple model to the initially 15 $M_{\odot}$ pre-supernova stellar model s15a28 [12].

Figure 1 shows $^{1}$ the time dependence of the temperature $T_{9}=T / 10^{9} \mathrm{~K}$ and mass density in two zones of s15a28 as computed with our simple SNII model with explosion energy $1.0 \times 10^{51}$ ergs. Zone 330 is at interior mass coordinate $1.63 M_{\odot}$, while zone 361 is a little farther out at interior mass coordinate $1.78 M_{\odot}$. In the calculations, the shock is generated at time $t=0$ at the innermost ejected zone. It reaches zone 330 at about 0.2 seconds and zone 361 at about 0.3 seconds. Before $\sim 0.2$ seconds, zone 330 is at its pre-shock temperature and density. When the shock hits zone 330, the temperature and density both rise suddenly as the zone is compressed by the shock. Once the shock passes, the matter expands and cools. Since zone 361 is farther out in the star, the shock arrives later. That zone also experiences a sudden rise in temperature and density, but the values attained are lower because the post-shock volume is now larger. Since the radiation energy density is proportional to $T^{4}$ and the explosion energy (in our assumption of a radiation-dominated, isothermal post-shock ball) is the energy density times the shocked volume, larger shocked volume means a lower post-shock temperature.

Figure 2 shows the time dependence of the mass fractions of key species in zones 330 and 361. Both zones are ${ }^{28} \mathrm{Si}$ rich in the pre-explosion star. Once the shock passes through the zones and the matter is compressed and heated, silicon burning converts the bulk of the pre-shock matter into ${ }^{56} \mathrm{Ni}$. The difference between zones 330 and 361 is that zone 330 experiences a high enough post-shock temperature to disintegrate a substantial number of heavy nuclei into alpha particles and free nucleons before reassembling them into heavy species. Incomplete reassembly of the light particles leads to an "alpha-rich freezeout". By contrast, in zone 361, the peak temperature is lower and leads to less disintegration. All of the light particles capture on remaining heavy nuclei to move

\footnotetext{
${ }^{1}$ Interested readers can find instructions for reproducing our explosion and network calculations at http://sourceforge.net/p/nucnet-projects/wiki/simple_snII/.
} 


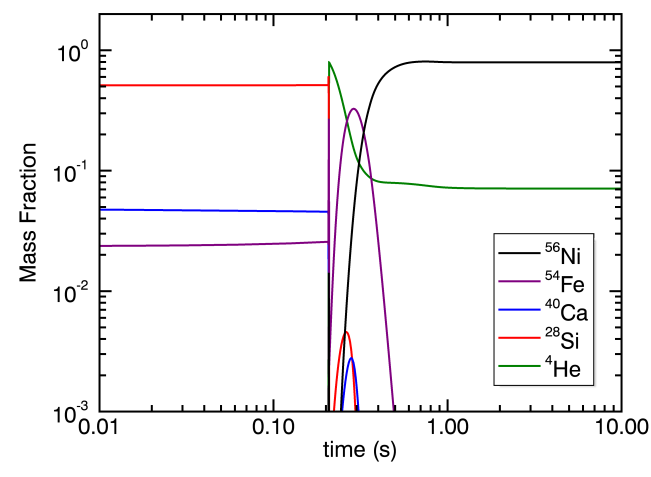

(a)

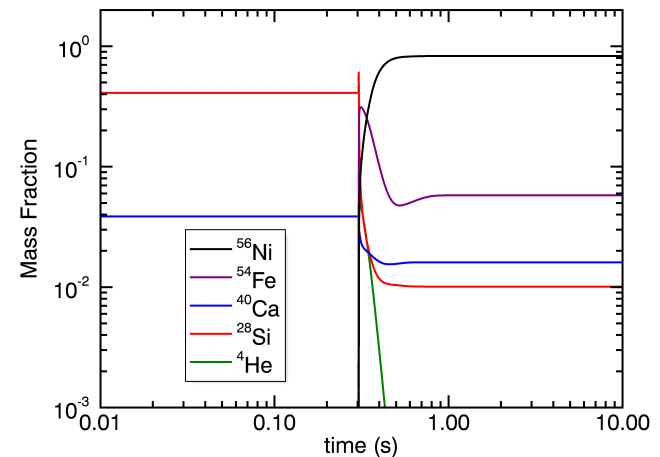

(b)

Figure 2: Time dependence of the mass fractions of key species in the simple SNII model in (a) zone 330 and (b) zone 330 .

them to higher mass and results in an "alpha-poor freezeout". In either case, however, the net result is that most ${ }^{28} \mathrm{Si}$ burns to ${ }^{56} \mathrm{Ni} .{ }^{2}$

Because the burning is rapid, weak decays do not have time to occur and alter the overall neutron-to-proton ratio in the matter. In fact, silicon burning proceeds through a quasi-equilibrium, which, owing to the negligible neutron excess present, is dominated by alpha $(Z=N)$ nuclei $[13$, $14,15]$, especially ${ }^{56} \mathrm{Ni}$. Days and months after shock passage, the ${ }^{56} \mathrm{Ni}$ decays to ${ }^{56} \mathrm{Co}$ and then to ${ }^{56} \mathrm{Fe}$. These decays deposit energy into the overlying stellar mantle and activate the important supernova displays already mentioned.

\section{The Secondary Machine}

To analyze the secondary machine, we consider the flow of nuclear energies during silicon burning through use of a simple, but realistic liquid-drop model for the nuclear mass [16]. That model divides the nuclear mass into the usual volume, surface, and Coulomb components. It also includes nucleon pairing and treats shell phenomena as a bunched Fermi gas.

Figure 3(a) shows ${ }^{3}$ the total nuclear Coulomb energy per nucleon (nuclear Coulomb energy in each species weighted by network abundances per nucleon) during the silicon burning calculations presented in Figure 2. In zone 361, the nuclear Coulomb energy rises from its initial value as increasingly heavy nuclei (with higher charge) form, reaches a plateau at reaction freezeout, and then subsequently declines as radioactive alpha nuclei decay to their $(Z-2, A)$ daughters. In zone 330 , the nuclear Coulomb energy first rises as the abundance distribution shifts to heavier nuclei, then, as the temperature continues to rise, falls as the nuclei disintegrate into light particle, then rises again as the light particles reassemble into heavy species. The same decline in the nuclear Coulomb energy occurs at late times.

\footnotetext{
${ }^{2}$ Movies showing the time evolution of the elemental abundances for the network calculations accompany this paper.

${ }^{3}$ Interested readers can find instructions for reproducing our nuclear mass calculations at http://sourceforge.net/p/nucnet-projects/wiki/nuclear_mass/.
} 


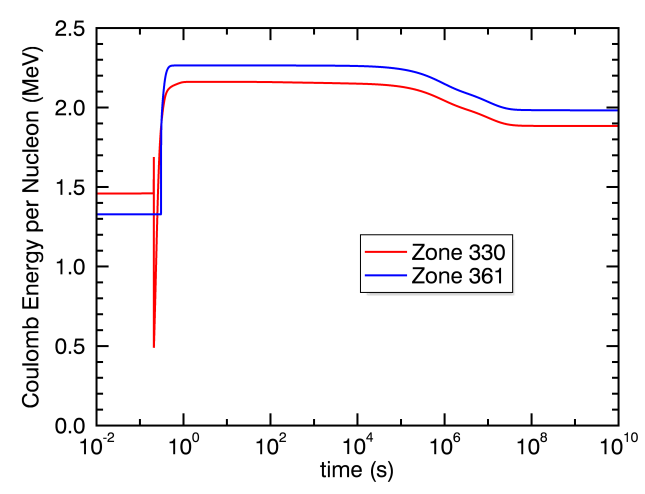

(a)

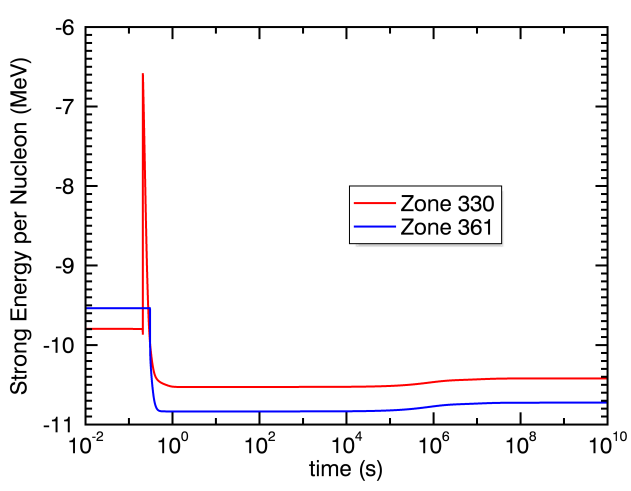

(b)

Figure 3: Time dependence of (a) the nuclear Coulomb energy and (b) the nuclear strong energy during the network calculations of silicon burning.

Figure 3(b) shows the strong nuclear energy per nucleon (the volume, surface, pairing, and shell terms in the nuclear mass weighted by network abundances) during the calculations. These curves show the complementary behavior to the Coulomb energy curves; thus, while Coulomb energy increases in the nuclei during the burning, the strong energy decreases (becomes more negative). In the mass model under study, the strong energy decrease largely results from the fact that nuclei become larger so that the fraction of the nucleons on the surface, which do not feel the full binding of the nuclear force, drops. This strong energy decline during silicon burning more than compensates the Coulomb energy increase and allows the overall burning to be exothermic. Significantly, the energy released by the burning is delivered promptly to the surrounding medium and, since it is but a small fraction of the local thermal energy content, it simply thermalizes without activating any important supernova displays.

As Coulomb energy builds up in alpha nuclei during rapid silicon burning, the Coulomb energy difference between those parent nuclei and their ultimate $(Z-2, A)$ daughters grows as $\sim Z^{2 / 3}$. Since alpha nuclei with $Z>20$ are radioactive, this Coulomb energy difference must eventually move to other forms. Not all of it can be radiated, however. Some must accommodate the increase in the mass of the two protons that must convert into neutrons, and some must accommodate the increase in the strong nuclear energy component of the nucleus (the small, late rise in the strong nuclear energy component seen in Fig. 3(b)). The remainder, which we call excess Coulomb energy, can be radiated. Of the $\sim 0.28 \mathrm{MeV}$ per nucleon decline in the Coulomb energy seen in Fig. 3(a), $0.03 \mathrm{MeV}$ per nucleon goes into changing two protons into heavier neutrons and $\sim 0.11 \mathrm{MeV}$ per nucleon goes into increasing the strong energy content of the nuclei; thus, $\sim 0.14$ $\mathrm{MeV}$ per nucleon is excess Coulomb energy that can be radiated.

Here then is the picture of the secondary machine. Gravitationally caused compression of the silicon shell ignites silicon burning which increases the Coulomb energy in nuclei. Excess of this stored Coulomb energy is later released. The portion of the released excess Coulomb energy not going to neutrinos is thus delivered to the supernova mantle as nonthermal energy that can activate supernova displays. 


\section{Conclusion}

Many astronomers and astrophysics simply but usefully understand core-collapse supernovae in terms of what we call the primary supernova machine: gravitational collapse $\rightarrow$ generation of the outward-moving shock $\rightarrow$ expulsion of the stellar mantle. In the same spirit, we add the notion of the secondary supernova machine: gravitational collapse $\rightarrow$ compression of the silicon zone $\rightarrow$ increase of Coulomb energy in nuclei $\rightarrow$ later release of excess of that Coulomb energy $\rightarrow$ astronomical displays. Readers may find this a simple, but useful way of picturing how energy stored early in the stellar explosion can be released later with significant consequences for astronomy.

\section{References}

[1] D. D. Clayton, S. A. Colgate, G. J. Fishman, Gamma-Ray Lines from Young Supernova Remnants, Astrophys. J. 155 (1969) 75.

[2] N. Gehrels, G. H. Share (Eds.), Nuclear spectroscopy of astrophysical sources, Vol. 170 of American Institute of Physics Conference Series, 1988.

[3] R. Sunyaev et al., E. Churazov, Discovery of hard X-ray emission from supernova 1987A, Nature 330 (1987) 227.

[4] T. Dotani, K. Hayashida, H. Inoue, M. Itoh, K. Koyama, Discovery of an unusual hard X-ray source in the region of supernova 1987A, Nature 330 (1987) 230.

[5] J. R. Graham, Radioactive ionization of the envelope of SN 1987A, Astrophys. J. Lett. 335 (1988) L53.

[6] T. Yu, B. S. Meyer, D. D. Clayton, Formation of $\mathrm{C}_{n}$ Molecules in Oxygen-rich Interiors of Type II Supernovae, Astrophys. J. 769 (2013) 38.

[7] W. D. Arnett, J. N. Bahcall, R. P. Kirshner, S. E. Woosley, Supernova 1987A, Ann. Rev. Astron. Astrophys. 27 (1989) 629.

[8] S. E. Woosley, T. A. Weaver, The physics of supernova explosions, Ann. Rev. Astron. Astrophys.24 (1986) 205.

[9] A. Burrows, Supernova explosions in the Universe, Nature 403 (2000) 727.

[10] M. J. Bojazi, B. S. Meyer, Explosive nucleosynthesis of N15 in a massive-star model, Phys. Rev. C 89 (2014) 025807

[11] S. E. Woosley, T. A. Weaver, The Evolution and Explosion of Massive Stars. II. Explosive Hydrodynamics and Nucleosynthesis, Astrophys. J. Suppl. 101 (1995) 181.

[12] T. Rauscher, A. Heger, R. D. Hoffman, S. E. Woosley, Nucleosynthesis in Massive Stars with Improved Nuclear and Stellar Physics, Astrophys. J. 576 (2002) 323.

[13] D. Bodansky, D. D. Clayton, W. A. Fowler, Nuclear Quasi-Equilibrium during Silicon Burning, Astrophys. J. Suppl. 16 (1968) 299.

[14] S. E. Woosley, W. D. Arnett, D. D. Clayton, The Explosive Burning of Oxygen and Silicon, Astrophys. J. Suppl. 26 (1973) 231.

[15] B. S. Meyer, T. D. Krishnan, D. D. Clayton, Theory of quasi-equilibrium nucleosynthesis and applications to matter expanding from high temperature and density, Astrophys. J. 498 (1998) 808.

[16] W. D. Myers, W. J. Swiatecki, Nuclear masses and deformations, Nucl. Phys. A 81 (1966) 1. 